\title{
New Construction of Fuzzy Soft Lattices
}

\author{
Dr. M. Marudai \\ Associate Professor, \\ Department of Mathematics \\ Bharathidasan University, \\ Tiruchirappalli -24 . \\ Tamilnadu. India
}

\author{
V. Rajendran \\ Research Scholar, \\ Department of Mathematics \\ Bharathidasan University, \\ Tiruchirappalli -24 . \\ Tamilnadu. India
}

\begin{abstract}
In this paper, we study Moldtsov notion of soft set and fuzzy soft set considering the fact that the parameters are mostly fuzzy hedges or fuzzy parameters. We also, study the notion of fuzzy soft lattice on groups, homomorphic image, preimage of fuzzy soft lattices, arbitrary family of fuzzy soft lattices and fuzzy normal soft lattices using T-norms. Also the notion of sensible fuzzy soft lattice in groups and some related properties on it are investigated.
\end{abstract}

Key Words: Soft set, Soft lattice, fuzzy soft lattice, Sensible fuzzy normal lattice, direct product.

\section{INTRODUCTION}

In 1999 Molodtsov [9], initiated the novel concept of soft set theory which is completely new approach for modeling, vagueness and uncertainties. Soft set theory has a rich potential for applications in several directions, few of which had been shown by Molodtsov in [9]. After Molodtsov work, some different applications of soft set theory were studied in [5].

Furthermore Maji, Biswas and Roy worked on soft set theory in [7]. Also Maji et.al [6] presented the definition of fuzzy soft set and Roy et.al presented some applications of thenotion in decision making problems. The algebraic structures of set theories dealing with uncertainities has also been studied by some authors. Rosenfield [10] proposed the concept of fuzzy groups in order to establish the algebraic structures of fuzzy sets. Rough groups were defined by Biswas and some authors have studied the algebraic properties of rough sets as well. Recently many authors discuss the soft set research on the soft set theory is progressing rapidly. For example, the concept of soft semi ring [4], soft groups [5], soft BCK / BCI algebras, soft BL-algebras and fuzzy soft groups.

In this paper, we study Moldtsov notion of soft set and fuzzy soft set considering the fact that the parameters are mostly fuzzy hedges or fuzzy parameters. We introduce the notion of fuzzy soft lattice on groups, homomorphic image, pre-image of fuzzy soft lattices, arbitrary family of fuzy soft lattices and fuzzy normal soft lattices using T-norms. Also the notion of sensible fuzzy soft lattices in groups and some related properties on it are investigated.

\section{PRELIMINARIES}

Definition 2.1 :A pair (f, A) is called a soft set over the lattice $\mathrm{L}$, if $\mathrm{f}: \mathrm{A} \square \mathrm{p}(\mathrm{L})$. Here $\mathrm{L}$ be the initial universe and $\mathrm{E}$ be the set of parameters. Let $\mathrm{p}(\mathrm{L})$ denotes the power set of $\mathrm{L}$ and IL denotes the set of all fuzzy set on L.

Definition 2.2:Let $M$ : (f, A) be a soft set and f: A $\square$ p(L) is a mapping. Where $L$ is a complete lattie then the soft set $M$ is called soft lattice.

Definition 2.3: A pair (f, A) is called a fuzzy soft set over L. Where f:A $\square$ IL, (ie) for each a $\square$ A, f: : L $\square$ I is a fuzzy set in $\mathrm{L}$.

Definition 2.4: Let (f, A) be a fuzzy soft set and $\mathrm{f}_{\mathrm{a}}$ : $\mathrm{A} \rightarrow$ $\mathrm{G}$ is called fuzzy soft lattice if

$$
\begin{aligned}
& \text { (FSL1) } \quad \mathrm{f}_{\mathrm{a}}(\mathrm{x}+\mathrm{y}) \quad \geq \mathrm{T}\left\{\mathrm{f}_{\mathrm{a}}(\mathrm{x}), \mathrm{f}_{\mathrm{a}}(\mathrm{y})\right\} \\
& \text { (FSL2) } \quad \mathrm{f}_{\mathrm{a}}(-\mathrm{x}) \quad \geq \mathrm{f}_{\mathrm{a}}(\mathrm{x}) \\
& \text { (FSL3) } \mathrm{f}_{\mathrm{a}}(\mathrm{x} \vee \mathrm{y}) \geq \mathrm{T}\left\{\mathrm{f}_{\mathrm{a}}(\mathrm{x}), \mathrm{f}_{\mathrm{a}}(\mathrm{y})\right\} \\
& \text { (FSL4) } \quad \mathrm{f}_{\mathrm{a}}(\mathrm{x} \Lambda \mathrm{y}) \geq \mathrm{T}\left\{\mathrm{f}_{\mathrm{a}}(\mathrm{x}), \mathrm{f}_{\mathrm{a}}(\mathrm{y})\right\} \quad \text { for all } \mathrm{x}, \mathrm{y} \in \mathrm{G}
\end{aligned}
$$

Definition 2.5 : Let $A$ be a fuzzy set and $f_{a}$ : $A \rightarrow G$ be a lattice ordered group of $A$ then $f_{a}$ is called fuzzy soft lattice ordered group (FSLG) If

$$
\begin{aligned}
& \left(\text { FSLG1) } f_{a}(x+y) \geq T\left\{f_{a}(x), f_{a}(y)\right\}\right. \\
& \left(\text { FSLG2) } f_{a}(-x) \geq f_{a}(x)\right. \\
& \left(\text { FSLG3) } f_{a}(0) \quad=1, \text { for all } x, y \in G\right.
\end{aligned}
$$

Definition 2.6 : By a t-norm $\mathrm{T}$, we mean a function $\mathrm{T}$ : $[0,1] \times[0,1] \rightarrow[0,1]$ satisfying the following conditions

(T4) $\mathrm{T}(\mathrm{x}, \mathrm{T}(\mathrm{y}, \mathrm{z}))=\mathrm{T}(\mathrm{T}(\mathrm{x}, \mathrm{y}), \mathrm{z}), \quad$ for all $\mathrm{x}, \mathrm{y}, \mathrm{z} \in[0$, 1]

Definition 2.7: For any fuzzy soft set $\mathrm{f}_{\mathrm{a}}$ in $\mathrm{G}$ and $\mathrm{t} \in[0$, $1]$, we define the set

$U\left(f_{a}: t\right)=\left\{x \in G / f_{a}(x) \geq t\right\}$, which is called an upper cut of $f_{a}$ and can be used to the characterization of $\mathrm{f}_{\mathrm{a}}$.

Definition 2.8: Let $f: G \rightarrow G^{\prime}$ be a lattice group homomorphism and $A$ be a fuzzy soft lattice of $G^{\prime}$ then $A$ $\mathrm{f}(\mathrm{x})=(\mathrm{A}$ of $)(\mathrm{x})=\mathrm{f}^{-1}(\mathrm{~A})(\mathrm{x})$

Definition 2.9 :Let $\theta: X \rightarrow Y$ be a map. A and B are fuzzy soft lattices in $\mathrm{X}$ and $\mathrm{Y}$ respectively. Then the inverse image of $\mathrm{B}$ under $\theta$ is a fuzzy soft set defined by 
$\left(\mathrm{f}_{\mathrm{a}}\right)_{\mathrm{B}}(\theta(\mathrm{x}))$

$\theta^{-1}(\mathrm{~B})=\left(\mathrm{f}_{\mathrm{a}}\right) \theta^{-1}(B)$ where $\left(\mathrm{f}_{\mathrm{a}}\right)_{\theta^{-1}(B)}(\mathrm{x})=$

Definition 2.10:Let $\mathrm{G}$ be a group and $\mathrm{A}$ be a fuzzy soft lattice of G, then A is called Fuzzy Normal soft lattice (FNSL) if $A(x y)=A(y x)$, for all $x, y \in G$

Definition 2.11: Let $\left(f_{a}\right)_{A}$ be a fuzzy soft set of G. Let $\theta: G \rightarrow G^{\prime}$ be a map. Define the map $\left(f_{a}\right)_{A}(\theta): G \rightarrow[0,1]$ by $\left(\mathrm{f}_{\mathrm{a}}\right)_{A^{\theta}}(\mathrm{x})=\left(\mathrm{f}_{\mathrm{a}}\right)_{\mathrm{A}}(\theta(\mathrm{x}))$.

\section{CHARATERIZATION OF FUZZY SOFT LATTICES}

Proposition 3.1 : Let $G$ and $G^{\prime}$ be two Fuzzy Soft Lattice ordered group and $\theta: \mathrm{G} \rightarrow \mathrm{G}^{\prime}$ be a homomorphism If $\mathrm{B}$ is a Fuzzy Soft Lattice of $\mathrm{G}^{\prime}$ then the pre-image $\theta^{-1}(\mathrm{~B})$ is Fuzzy Soft Lattice of G.

Proof : Assume that B is a Fuzzy Soft Lattice of G. Let x, y $\in \mathrm{G}$.

$$
\begin{gathered}
(\mathrm{FSL} 1)\left(\mathrm{f}_{\mathrm{a}}\right)_{\theta^{-1}(B)}(\mathrm{x}+\mathrm{y})=\left(\mathrm{f}_{\mathrm{a}}\right)_{\mathrm{B}} \theta(\mathrm{x}+\mathrm{y}) \\
=\left(\mathrm{f}_{\mathrm{a}}\right)_{\mathrm{B}}(\theta(\mathrm{x})+\theta(\mathrm{y})) \\
\geq \mathrm{T}\left\{\left(\mathrm{f}_{\mathrm{a}}\right)_{\mathrm{B}}\left(\theta(\mathrm{x}),\left(\mathrm{f}_{\mathrm{a}}\right)_{\mathrm{B}} \theta(\mathrm{y})\right)\right\} \\
\geq \mathrm{T}\left\{\left(\mathrm{f}_{\mathrm{a}}\right)_{\theta^{-1}(B)}(\mathrm{x}),\left(\mathrm{f}_{\mathrm{a}}\right)_{\theta^{-1}(B)}(\mathrm{y})\right\} \\
(\mathrm{FSL} 2)\left(\mathrm{f}_{\mathrm{a}}\right)_{\theta^{-1}(B)}^{(-\mathrm{x}) \quad} \quad\left(\mathrm{f}_{\mathrm{a}}\right)_{\mathrm{B}}(\theta(-\mathrm{x})) \\
\quad \geq\left(\mathrm{f}_{\mathrm{a}}\right)_{\mathrm{B}}(\theta(\mathrm{x})) \\
\geq\left(\mathrm{f}_{\mathrm{a}}\right)_{\theta^{-1}(B)}(\mathrm{x})
\end{gathered}
$$

$(\mathrm{FSL3})\left(\mathrm{f}_{\mathrm{a}}\right) \theta_{\theta^{-1}(B)}(\mathrm{x} \mathrm{Vy})=\left(\mathrm{f}_{\mathrm{a}}\right)_{\mathrm{B}} \theta(\mathrm{x} \mathrm{V} \mathrm{y})$

$$
=\left(\mathrm{f}_{\mathrm{a}}\right)_{\mathrm{B}}(\theta \mathrm{x} \mathrm{V} \theta \mathrm{y})
$$

$$
\geq \mathrm{T}\left\{\left(\mathrm{f}_{\mathrm{a}}\right)_{\mathrm{B}}(\theta(\mathrm{x})),\left(\mathrm{f}_{\mathrm{a}}\right)_{\mathrm{B}}(\theta(\mathrm{y}))\right\}
$$

$$
\geq \mathrm{T}\left\{\left(\mathrm{f}_{\mathrm{a}}\right)_{\theta^{-1}(B)}(\mathrm{x}),\left(\mathrm{f}_{\mathrm{a}}\right) \theta^{-1}(B)(\mathrm{y})\right\}
$$

$(\mathrm{FSL} 4)\left(\mathrm{f}_{\mathrm{a}}\right)_{\theta^{-1}(B)}\left(\mathrm{x}^{\wedge} \mathrm{y}\right)=\left(\mathrm{f}_{\mathrm{a}}\right)_{\mathrm{B}} \theta\left(\mathrm{x}^{\wedge} \mathrm{y}\right)$

$$
\begin{aligned}
&=\left(\mathrm{f}_{\mathrm{a}}\right)_{\mathrm{B}}\left(\theta \mathrm{x}^{\wedge} \theta \mathrm{y}\right) \\
& \geq \mathrm{T}\left\{\left(\mathrm{f}_{\mathrm{a}}\right)_{\mathrm{B}}(\theta(\mathrm{x})), \mu_{\mathrm{B}}(\theta(\mathrm{y})\}\right. \\
& \geq \mathrm{T}\left\{\left(\mathrm{f}_{\mathrm{a}}\right)_{\theta^{-1}(B)}(\mathrm{x}),\left(\mathrm{f}_{\mathrm{a}}\right)_{\theta^{-1}(B)}(\mathrm{y})\right\}
\end{aligned}
$$

$\therefore \theta^{-1}(\mathrm{~B})$ is a Fuzzy Soft Lattice of G.

Proposition 3.2 : Let $\theta: G \rightarrow G^{\prime}$ be an epimorphism and B is a Fuzzysoft set in $G^{\prime}$. If $\theta^{-1}(B)$ is Fuzzy Soft Lattice of $G$ then B is Fuzzy Soft Lattice of $\mathrm{G}^{\prime}$.

Proof : Let $x, y \in G$, there exist an element $a, b \in G^{\prime}$ such that $\theta(\mathrm{a})=\mathrm{x}, \quad \theta(\mathrm{b})=\mathrm{y}$

$\left(\right.$ FSL1) $\left(f_{a}\right)_{B}(x+y)=\left(f_{a}\right)_{B}(\theta(a)+\theta(b))$

$$
=\left(f_{a}\right)_{B} \theta(a+b)
$$

$$
\begin{aligned}
& =\left(\mathrm{f}_{\mathrm{a}}\right)_{\theta^{-1}(B)}(\mathrm{a}+\mathrm{b}) \\
& \geq \mathrm{T}\left\{\left(\mathrm{f}_{\mathrm{a}}\right)_{\theta^{-1}(B)}(\mathrm{a}),\left(\mathrm{f}_{\mathrm{a}}\right)_{\theta^{-1}(B)}^{(\mathrm{b})\}}\right. \\
& \geq \mathrm{T}\left\{\left(\mathrm{f}_{\mathrm{a}}\right)_{\mathrm{B}} \theta(\mathrm{a}),\left(\mathrm{f}_{\mathrm{a}}\right)_{\mathrm{B}} \theta(\mathrm{b})\right\} \\
& \geq \mathrm{T}\left\{\left(\mathrm{f}_{\mathrm{a}}\right)_{\mathrm{B}}(\mathrm{x}),\left(\mathrm{f}_{\mathrm{a}}\right)_{\mathrm{B}}(\mathrm{y})\right\} \\
& \left(\text { FSL2) }\left(f_{a_{B}}\right)_{B}(-x)=\left(f_{a}\right)_{B}(-\theta(a))\right. \\
& =\left(\mathrm{f}_{\mathrm{a}}\right)_{\mathrm{B}}(\theta(-\mathrm{a})) \\
& =\left(\mathrm{f}_{\mathrm{a}}\right) \theta^{-1}(B) \stackrel{(-\mathrm{a})}{ } \\
& \geq\left(\mathrm{f}_{\mathrm{a}}\right)_{\theta^{-1}(B)} \\
& \geq\left(\mathrm{f}_{\mathrm{a}}\right)_{(B)} \theta(\mathrm{a}) \\
& \geq\left(\mathrm{f}_{\mathrm{a}}\right)_{(B)}(\mathrm{x}) \\
& =\left(f_{a}\right)_{B} \theta(a V b) \\
& =\left(\mathrm{f}_{\mathrm{a}}\right) \theta^{-1}(B)(\mathrm{a} \mathrm{V} \mathrm{b}) \\
& \geq \mathrm{T}\left\{\left(\mathrm{f}_{\mathrm{a}}\right)_{\theta^{-1}(B)}{ }^{(\mathrm{a}),\left(\mathrm{f}_{\mathrm{a}}\right)} \theta^{-1}(B){ }^{(\mathrm{b})}\right\} \\
& \geq \mathrm{T}\left\{\left(\mathrm{f}_{\mathrm{a}}\right)_{\mathrm{B}} \theta(\mathrm{a}),\left(\mathrm{f}_{\mathrm{a}}\right)_{\mathrm{B}} \theta(\mathrm{b})\right\} \\
& \geq \mathrm{T}\left\{\left(\mathrm{f}_{\mathrm{a}}\right)_{\mathrm{B}}(\mathrm{x}),\left(\mathrm{f}_{\mathrm{a}}\right)_{\mathrm{B}}(\mathrm{y})\right\} \\
& \left(\text { FSL4) }\left(f_{a}\right)_{B}(x \Lambda y)=\left(f_{a}\right)_{B}(\theta(a) \Lambda \theta(b))\right. \\
& =\left(f_{\mathrm{a}}\right)_{\mathrm{B}}(\theta(\mathrm{a} \Lambda \mathrm{b})) \\
& =\left(\mathrm{f}_{\mathrm{a}}\right) \theta^{-1}(B)(\mathrm{a} \Lambda \mathrm{b}) \\
& \geq \mathrm{T}\left\{\left(\mathrm{f}_{\mathrm{a}}\right)_{\theta^{-1}(B)}(\mathrm{a}),\left(\mathrm{f}_{\mathrm{a}}\right)_{\theta^{-1}(B)}(\mathrm{b})\right\} \\
& \geq \mathrm{T}\left\{\left(\mathrm{f}_{\mathrm{a}}\right)_{\mathrm{B}} \theta(\mathrm{a}),\left(\mathrm{f}_{\mathrm{a}}\right)_{\mathrm{B}} \theta(\mathrm{b})\right\} \\
& \geq \mathrm{T}\left\{\left(\mathrm{f}_{\mathrm{a}}\right)_{\mathrm{B}}(\mathrm{x}),\left(\mathrm{f}_{\mathrm{a}}\right)_{\mathrm{B}}(\mathrm{y})\right\}
\end{aligned}
$$

$\therefore \mathrm{B}$ is a Fuzzy Soft Lattice of $\mathrm{G}^{\prime}$.

Proposition 3.3 : If $\left\{\mathrm{A}_{\mathrm{i}}\right\}$ is a family of Fuzzy Soft Lattice of $G$ then $\cap A_{i}$ is a Fuzzy Soft Lattice of G. Where $\cap \mathrm{A}_{\mathrm{I}}=\left\{\mathrm{x}, \Lambda\left(\mathrm{f}_{\mathrm{a}}\right)_{A_{i}}(\mathrm{x}) / \mathrm{x} \in \mathrm{G}\right\}$, here $\mathrm{i} \in \mathrm{A}$.

Proof : Let $x, y \in G$ then for $i \in A$ it follows that

$$
\begin{array}{r}
(\mathrm{FSL} 1)\left(\cap\left(\mathrm{f}_{\mathrm{a}}\right)_{A_{i}}\right)(\mathrm{x}+\mathrm{y})=\Lambda\left(\mathrm{f}_{\mathrm{a}}\right)_{A_{i}}(\mathrm{x}+\mathrm{y}) \\
\geq \Lambda \mathrm{T}\left\{\left(\mathrm{f}_{\mathrm{a}}\right)_{A_{i}}(\mathrm{x}),\left(\mathrm{f}_{\mathrm{a}}\right)_{A_{i}}(\mathrm{y})\right\} \\
\geq \Lambda \mathrm{T}\left\{\left(\cap\left(\mathrm{f}_{\mathrm{a}}\right)_{A_{i}}\right)(\mathrm{x}),\left(\cap\left(\mathrm{f}_{\mathrm{a}}\right)_{A_{i}}\right)(\mathrm{y})\right\} \\
(\mathrm{FL} 2)\left(\cap\left(\mathrm{f}_{\mathrm{a}}\right)_{A_{i}}\right)(-\mathrm{x}) \quad \Lambda\left(\mathrm{f}_{\mathrm{a}}\right)_{A_{i}}(-\mathrm{x}) \\
\geq \Lambda\left(\mathrm{f}_{\mathrm{a}}\right)_{A_{i}}(\mathrm{x}) \\
\geq\left(\cap\left(\mathrm{f}_{\mathrm{a}}\right)_{A_{i}}\right)(\mathrm{x})
\end{array}
$$




$$
\begin{aligned}
& (\text { FL3 })\left(\cap\left(\mathrm{f}_{\mathrm{a}}\right)_{A_{i}}\right)(\mathrm{x} \vee \mathrm{y})=\Lambda\left(\mathrm{f}_{\mathrm{a}}\right)_{A_{i}}(\mathrm{x} \vee \mathrm{y}) \\
& \geq \Lambda \mathrm{T}\left\{\left(\cap\left(\mathrm{f}_{\mathrm{a}}\right)_{A_{i}}\right)(\mathrm{x}),\left(\cap\left(\mathrm{f}_{\mathrm{a}}\right)_{A_{i}}\right)(\mathrm{y})\right\} \\
& \geq \mathrm{T}\left\{\left(\cap\left(\mathrm{f}_{\mathrm{a}}\right)_{A_{i}}\right)(\mathrm{x}),\left(\cap\left(\mathrm{f}_{\mathrm{a}}\right)_{A_{i}}\right)(\mathrm{y})\right\} \\
& (\text { FL4 })\left(\cap\left(\mathrm{f}_{\mathrm{a}}\right)_{A_{i}}\right)(\mathrm{x} \Lambda \mathrm{y})=\Lambda\left(\mathrm{f}_{\mathrm{a}}\right)_{A_{i}}(\mathrm{x} \Lambda \mathrm{y}) \\
& \geq \Lambda \mathrm{T}\left\{\left(\mathrm{f}_{\mathrm{a}}\right)_{A_{i}}(\mathrm{x}),\left(\mathrm{f}_{\mathrm{a}}\right)_{A_{i}}(\mathrm{y})\right\} \\
& \geq \mathrm{T}\left\{\left(\cap\left(\mathrm{f}_{\mathrm{a}}\right)_{A_{i}}\right)(\mathrm{x}),\left(\cap\left(\mathrm{f}_{\mathrm{a}}\right)_{A_{i}}\right)(\mathrm{y})\right\}
\end{aligned}
$$

$\therefore \cap \mathrm{A}_{\mathrm{i}}$ is a Fuzzy Soft Lattice of G.

Proposition 3.4 :If A is Fuzzy Soft set in G such that all non empty level subject $U(A ; t)$ is a Fuzzy Soft Lattice of $G$ then A is Fuzzy Soft Lattice of G.

Proof : Let $x, y \in U(A ; t)$, we have $A(x) \geq t$ and $A(y) \geq t$. So that $x+y \in U(A ; t)$ we have $A(x+y) \geq t$.

(FSL1) $A(x+y) \geq t \geq \min \{t, t\}$

$\geq \mathrm{T}\{\mathrm{A}(\mathrm{x}), \mathrm{A}(\mathrm{y})\}$

(FSL2) $\mathrm{A}(-\mathrm{x}) \quad \geq \mathrm{t}=\mathrm{A}(\mathrm{x}) \geq \mathrm{A}(\mathrm{x})$

(FSL3) $\mathrm{A}(\mathrm{x} V \mathrm{y}) \geq \mathrm{t} \geq \min \{\mathrm{t}, \mathrm{t}\} \geq \mathrm{T}\{\mathrm{A}(\mathrm{x}), \mathrm{A}(\mathrm{y})\}$

(FSL4) A (x $\Lambda \mathrm{y}) \geq \mathrm{t} \geq \min \{\mathrm{t}, \mathrm{t}\} \geq \mathrm{T}\{\mathrm{A}(\mathrm{x}), \mathrm{B}(\mathrm{y})\}$

$\therefore A$ is a Fuzzy Soft Lattice of G.

Proposition 3. :Let A be a Fuzzy Soft Lattice of G. Let $\mathrm{A}^{\oplus}$ be a Fuzzy set in $\mathrm{G}$ defined by $\mathrm{A}^{\oplus}(\mathrm{x})=\mathrm{A}(\mathrm{x})+1-\mathrm{A}(0)$, for all $x \in G$. then $A^{\oplus}$ is Normal Fuzzy Soft Lattice of $G$ which contains A.

Proof : For any $x \in G$,

$$
\mathrm{A}^{\oplus}=\mathrm{A}(\mathrm{x})+1-\mathrm{A}(0)
$$

(FSL1) $\mathrm{A}^{\oplus}(\mathrm{x}+\mathrm{y})$

$=\mathrm{A}(\mathrm{x}+\mathrm{y})+1-\mathrm{A}(0)$

$\geq \mathrm{T}\{\mathrm{A}(\mathrm{x}), \mathrm{A}(\mathrm{y})\}+1-\mathrm{A}(0)$

$\geq \mathrm{T}\{\mathrm{A}(\mathrm{x})+1-\mathrm{A}(0), \mathrm{A}(\mathrm{y})+1-\mathrm{A}(0)\}$

$\geq \mathrm{T}\left\{\mathrm{A}^{\oplus}(\mathrm{x}), \mathrm{A}^{\oplus}(\mathrm{y})\right\}$

$\left(\right.$ FSL2) $\mathrm{A}^{\oplus}(-\mathrm{x})=\mathrm{A}(-\mathrm{x})+1-\mathrm{A}(0)$

$$
\begin{aligned}
& \geq \mathrm{A}(\mathrm{x})+1-\mathrm{A}(0) \\
& \geq \mathrm{A}^{\oplus}(\mathrm{x})
\end{aligned}
$$

$(\mathrm{FSL} 3) \mathrm{A}^{\oplus}(\mathrm{x}$ V y $)=\mathrm{A}(\mathrm{x}$ V y $)+1-\mathrm{A}(0)$

$$
\begin{aligned}
& \geq \mathrm{T}\{\mathrm{A}(\mathrm{x}), \mathrm{A}(\mathrm{y})\}+1-\mathrm{A}(0) \\
& \geq \mathrm{T}\{\mathrm{A}(\mathrm{x})+1-\mathrm{A}(0), \mathrm{A}(\mathrm{y})+1-\mathrm{A}(0)\} \\
& \geq \mathrm{T}\left\{\mathrm{A}^{\oplus}(\mathrm{x}), \mathrm{A}^{\oplus}(\mathrm{y})\right\}
\end{aligned}
$$

(FSL4) $\mathrm{A}^{\oplus}(\mathrm{x} \Lambda \mathrm{y})=\mathrm{A}(\mathrm{x} \Lambda \mathrm{y})+1-\mathrm{A}(0)$

$$
\geq \mathrm{T}\{\mathrm{A}(\mathrm{x}), \mathrm{A}(\mathrm{y})\}+1-\mathrm{A}(0)
$$

$\geq \mathrm{T}\{\mathrm{A}(\mathrm{x})+1-\mathrm{A}(0), \mathrm{A}(\mathrm{y})+1-\mathrm{A}(0)\}$

$\geq \mathrm{T}\left\{\mathrm{A}^{\oplus}(\mathrm{x}), \mathrm{A}^{\oplus}(\mathrm{y})\right\}$

$\therefore \mathrm{A}^{\oplus}$ is a Fuzzy Soft Lattice of $\mathrm{G}$ and $\mathrm{A}^{\oplus} \mathrm{C} \mathrm{A}$.
Proposition 3.6 : If A is a Fuzzy Soft Lattice of G and $\theta$ is a homomorphism of $G$ than the Fuzzy Soft set $A^{\theta}$ of $G$ given by $A^{\theta}=\left\{\left\langle x ;\left(f_{a}\right) A^{\theta} \quad(x)>, x \in G\right\}\right.$ is a Fuzzy Soft Lattice of $\mathrm{G}$.

Proof : For any x, y G, we have

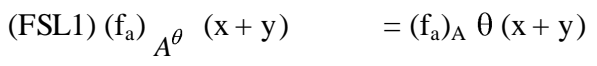

$$
\begin{aligned}
& =\left(\mathrm{f}_{\mathrm{a}}\right)_{\mathrm{A}}(\theta(\mathrm{x})+\theta(\mathrm{y})) \\
& \geq \mathrm{T}\left\{\left(\mathrm{f}_{\mathrm{a}}\right)_{\mathrm{A}} \theta(\mathrm{x}),\left(\mathrm{f}_{\mathrm{a}}\right)_{\mathrm{A}} \theta(\mathrm{y})\right\} \\
& \geq \mathrm{T}\left\{\left(\mathrm{f}_{\mathrm{a}}\right) A^{\theta}(\mathrm{x}),\left(\mathrm{f}_{\mathrm{a}}\right) A^{\theta}(\mathrm{y})\right\} \\
& \text { (FSL2) }\left(\mathrm{f}_{\mathrm{a}}\right) A^{\theta}(-\mathrm{x})=\left(\mathrm{f}_{\mathrm{a}}\right) A^{\theta}(-\mathrm{x}) \\
& \geq\left(\mathrm{f}_{\mathrm{a}}\right) A_{A^{\theta}}(\mathrm{x}) \\
& \text { (FSL3) } \mu_{A^{\theta}}(\mathrm{x} V \mathrm{y}) \quad=\left(\mathrm{f}_{\mathrm{a}}\right)_{\mathrm{A}} \theta(\mathrm{x} \mathrm{V} \mathrm{y}) \\
& =\left(\mathrm{f}_{\mathrm{a}}\right)_{\mathrm{A}}(\theta(\mathrm{x}) \mathrm{V} \theta(\mathrm{y})) \\
& \geq \mathrm{T}\left\{\left(\mathrm{f}_{\mathrm{a}}\right)_{\mathrm{A}} \theta(\mathrm{x}),\left(\mathrm{f}_{\mathrm{a}}\right)_{\mathrm{A}} \theta(\mathrm{y})\right\} \\
& \geq \mathrm{T}\left\{\left(\mathrm{f}_{\mathrm{a}}\right) A^{\theta}(\mathrm{x}),\left(\mathrm{f}_{\mathrm{a}}\right){ }_{A^{\theta}}(\mathrm{y})\right\}
\end{aligned}
$$

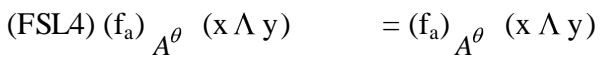

$$
\begin{aligned}
& \begin{array}{l}
=\left(\mathrm{f}_{\mathrm{a}}\right)_{\mathrm{A}}(\theta(\mathrm{x}) \Lambda \theta(\mathrm{y})) \\
\geq \mathrm{T}\left\{\left(\mathrm{f}_{\mathrm{a}}\right)_{\mathrm{A}} \theta(\mathrm{x}),\left(\mathrm{f}_{\mathrm{a}}\right)_{\mathrm{A}} \theta(\mathrm{y})\right\} \\
\geq \mathrm{T}\left\{\left(\mathrm{f}_{\mathrm{a}}\right)_{A^{\theta}}(\mathrm{x}),\left(\mathrm{f}_{\mathrm{a}}\right) A^{\theta}(\mathrm{y})\right\}
\end{array}
\end{aligned}
$$

$\therefore \mathrm{A}^{\theta}$ is a Fuzzy Soft Lattice of G.

Proposition 3.7 : Let $\mathrm{T}$ be a continuous t-norm and let $\mathrm{m}$ be a homomorphism on G. If $f_{a}$ is Fuzzy Soft Lattice of $G$ then $\left(f_{a}\right)^{m}$ is Fuzzy Soft Lattice of $f(G)$.

Proof : Let $A_{1}=f^{-1}\left(y_{1}\right), A_{2}=f^{-1}\left(y_{2}\right)$ and $A_{12}=f^{-1}\left(y_{1}+y_{2}\right)$. Where $\mathrm{y}_{1}+\mathrm{y}_{2} \in \mathrm{f}(\mathrm{G})$. Consider the set

$$
A_{1}+A_{2}=\left\{x \in G / x=a_{1}+a_{2}\right\}
$$

For some $\mathrm{a}_{1} \in \mathrm{A}_{1}$ and $\mathrm{a}_{2} \in \mathrm{A}_{2}$. If $\mathrm{x} \in \mathrm{A}_{1}+\mathrm{A}_{2}$ then $\mathrm{x}$ $=x_{1}+x_{2}$. So that, we have follows that

$$
\begin{aligned}
& \mathrm{f}(\mathrm{x}) \quad=\mathrm{f}\left(\mathrm{x}_{1}+\mathrm{x}_{2}\right)=\mathrm{f}\left(\mathrm{x}_{1}\right)+\mathrm{f}\left(\mathrm{x}_{2}\right)=\mathrm{y}_{1}+\mathrm{y}_{2} \\
& \text { So } \mathrm{x} \in \mathrm{f}^{-1}\left(\mathrm{y}_{1}+\mathrm{y}_{2}\right)=\mathrm{A}_{12}, \text { thus } A_{1}+A_{2} C A_{12} . \text { It }
\end{aligned}
$$

(FSL1) $\left(\mathrm{f}_{\mathrm{a}}\right)^{\mathrm{m}}\left(\mathrm{y}_{1}+\mathrm{y}_{2}\right)$

$=\sup \left\{\mathrm{f}_{\mathrm{a}}(\mathrm{x}) / \mathrm{x} \in \mathrm{f}^{-1}\left(\mathrm{y}_{1}+\mathrm{y}_{2}\right)\right\}$

$=\sup \left\{\mathrm{f}_{\mathrm{a}}(\mathrm{x}) / \mathrm{x} \in \mathrm{A}_{12}\right\}$

$\geq \sup \left\{\mathrm{f}_{\mathrm{a}}(\mathrm{x}) / \mathrm{x} \in \mathrm{A}_{1}+\mathrm{A}_{2}\right\}$

$\geq \sup \left\{f_{a}\left(x_{1}+x_{2}\right) / x_{1} \in A_{1}, x_{2} \in A_{2}\right\}$

$\geq \sup \left\{\mathrm{T}\left\{\mathrm{f}_{\mathrm{a}}\left(\mathrm{x}_{1}\right), \mathrm{f}_{\mathrm{a}}\left(\mathrm{x}_{2} / \mathrm{x}_{1} \in \mathrm{A}_{1}, \mathrm{x}_{2} \in \mathrm{A}_{2}\right\}\right\}\right.$

Since $\mathrm{T}$ is continuous for $\in>0$, We see that

If $\operatorname{Sup}\left\{\mathrm{f}_{\mathrm{a}}\left(\mathrm{x}_{1}\right) / \mathrm{x}_{1} \in \mathrm{A}_{1}\right\}+\mathrm{x}_{1} * \leq \delta$ and $\operatorname{Sup}\left\{\mathrm{f}_{\mathrm{a}}\left(\mathrm{x}_{2}\right) / \mathrm{x}_{2}\right.$ $\left.\in \mathrm{A}_{2}\right\}+\mathrm{x}_{2} * \leq \delta$ 
$T\left\{\sup \left\{f_{a}\left(x_{1}\right) x_{1} \in A_{1}\right\}, \operatorname{Sup}\left\{f_{a}\left(x_{2}\right) / x_{2} \in A_{2}\right\}\right\}+T\left(x_{1} *, x_{2}\right.$ $*) \leq$ ..... (1)

Choose $a_{1} \in A_{1}$ and $a_{2} \in A_{2}$, such that

$\operatorname{Sup}\left\{\mathrm{f}_{\mathrm{a}}\left(\mathrm{x}_{1}\right) / \mathrm{x}_{1} \in \mathrm{A}_{1}\right\}+\mathrm{f}_{\mathrm{a}}\left(\mathrm{a}_{1}\right) \leq \delta$ and $\operatorname{Sup}\left\{\mathrm{f}_{\mathrm{a}}\left(\mathrm{x}_{2}\right) / \mathrm{x}_{2}\right.$ $\left.\in \mathrm{A}_{2}\right\}+\mathrm{f}_{\mathrm{a}}\left(\mathrm{a}_{2}\right) \leq \delta$

Then

$\mathrm{T}\left\{\operatorname{Sup}\left\{\mathrm{f}_{\mathrm{a}}\left(\mathrm{x}_{1}\right) / \mathrm{x}_{1} \in \mathrm{A}_{1}\right\}, \operatorname{Sup}\left\{\mathrm{f}_{\mathrm{a}}\left(\mathrm{x}_{2}\right) / \mathrm{x}_{2} \in \mathrm{A}_{2}\right\}+\mathrm{T}\left(\mathrm{f}_{\mathrm{a}}\left(\mathrm{a}_{1}\right)\right.\right.$, $\left.f_{a}\left(a_{2}\right)\right) \leq \in \ldots(2)$

Thus we have

$\left(f_{a}\right)^{m}(x+y) \quad \geq \operatorname{Sup}\left\{T\left(f_{a}\left(x_{1}\right), f_{a}\left(x_{2}\right)\right) / x_{1} \in A_{1}, x_{2}\right.$ $\left.\in \mathrm{A}_{2}\right\}$

$=T\left\{\operatorname{Sup}\left(\mathrm{f}_{\mathrm{a}}\left(\mathrm{x}_{1}\right) / \mathrm{x}_{1} \in \mathrm{A}_{1}\right), \operatorname{Sup}\left(\mathrm{f}_{\mathrm{a}}\left(\mathrm{x}_{2}\right) / \mathrm{x}_{2}\right.\right.$ $\left.\left.\in \mathrm{A}_{2}\right)\right\} \geq \mathrm{T}\left\{\left(\mathrm{f}_{\mathrm{a}}\right)^{\mathrm{m}}(\mathrm{x}),\left(\mathrm{f}_{\mathrm{a}}\right)^{\mathrm{m}}(\mathrm{y})\right\}$

Similarly, we can show

$\left(\right.$ FSL2) $\quad\left(\mathrm{f}_{\mathrm{a}}\right)^{\mathrm{m}}(-\mathrm{x}) \geq\left(\mathrm{f}_{\mathrm{a}}\right)^{\mathrm{m}}(\mathrm{x})$

(FSL3) $\left(\mathrm{f}_{\mathrm{a}}\right)^{\mathrm{m}}(\mathrm{x}$ V y $) \quad \geq \mathrm{T}\left\{\left(\mathrm{f}_{\mathrm{a}}\right)^{\mathrm{m}}(\mathrm{x}),\left(\mathrm{f}_{\mathrm{a}}\right)^{\mathrm{m}}(\mathrm{y})\right\}$ and

$\left(\right.$ FSL4) $\left(\mathrm{f}_{\mathrm{a}}\right)^{\mathrm{m}}(\mathrm{x} \Lambda \mathrm{y}) \quad \geq \mathrm{T} \quad\left\{\left(\mathrm{f}_{\mathrm{a}}\right)^{\mathrm{m}}\right.$ $\left.\left(f_{a}\right)^{m}(y)\right\}$ Thus $\left(f_{a}\right)^{m}$ is fuzzy soft lattice of $G$.

Proposition 3.8 : Let $\mathrm{T}$ be a t-norm. Then every sensible Fuzzy Soft Lattice A of G is Fuzzy Soft Lattice of G.

Proof : Assume that A is sensible Fuzzy Soft Lattice of G then we have

(FSL1) $\mathrm{A}(\mathrm{x}+\mathrm{y}) \geq \mathrm{T}\{\mathrm{A}(\mathrm{x}), \mathrm{A}(\mathrm{y})\}$

(FSL2) $\mathrm{A}(-\mathrm{x}) \quad \geq \mathrm{A}(\mathrm{x})$

(FSL3) $\mathrm{A}(\mathrm{x}$ V y $) \geq \mathrm{T}\{\mathrm{A}(\mathrm{x}), \mathrm{A}(\mathrm{y})\}$

(FSL4)A $(\mathrm{x} \Lambda \mathrm{y}) \geq \mathrm{T}\{\mathrm{A}(\mathrm{x}), \mathrm{A}(\mathrm{y})\}, \quad$ for all $\mathrm{x}_{1} \mathrm{y} \in \mathrm{G}$.

Since A is sensible, we have

$\mathrm{T}\{\mathrm{A}(\mathrm{x}), \mathrm{A}(\mathrm{y})\}$

$=\mathrm{T}\{\{\mathrm{S}(\mathrm{A}(\mathrm{x}), \mathrm{A}(\mathrm{y})\},\{\mathrm{S}(\mathrm{A}(\mathrm{x}), \mathrm{A}(\mathrm{y})\}\}$

$\leq \mathrm{T}\{\mathrm{A}(\mathrm{x}), \mathrm{A}(\mathrm{y})\}$,

$\mathrm{T}(\mathrm{A}(\mathrm{x}), \mathrm{A}(\mathrm{y}))=\mathrm{T}\{\mathrm{A}(\mathrm{x}), \mathrm{A}(\mathrm{y})\}$

It follows that

$\mathrm{A}(\mathrm{x}+\mathrm{y}) \geq \mathrm{T}(\mathrm{A}(\mathrm{x}), \mathrm{A}(\mathrm{y}))$

$=\mathrm{T}\{\mathrm{A}(\mathrm{x}), \mathrm{A}(\mathrm{y})\}$, for all $\mathrm{x}_{1} \mathrm{y} \in \mathrm{G}$.

Similarly, we can show (FSL2), (FSL3) and (FSL4) of Definition (2.4)

So A is a Fuzzy Soft Lattice of G.

Proposition 3.9: An onto homomorphic image of Fuzzy Soft Lattice with sup property is Fuzzy Soft Lattice.

Proof : Let $f: G \rightarrow G^{\prime}$ be an onto homomorphism of $G$ and let A be Fuzzy Soft Lattice of $\mathrm{G}$ with sup property.

${ }^{1}\left(\mathrm{y}^{\prime}\right)$ be such that

Given $x, y \in G$, we let $x_{o} \in f^{-1}\left(x^{\prime}\right)$ and $y_{o} \in f^{-}$

$$
\begin{array}{rc}
A\left(x_{0}\right)=\operatorname{Sup~A}(h), & A\left(y_{0}\right)=\operatorname{Sup~A(h)} \\
h \in \mathrm{f}^{-1}\left(\mathrm{x}^{\prime}\right) & \mathrm{h} \in \mathrm{f}^{-1}\left(\mathrm{y}^{\prime}\right)
\end{array}
$$

Respectively. Then we can deduce that
(FSL1) $A^{f}\left(x^{\prime}+y^{\prime}\right)=\operatorname{Sup} A(z)$

$$
\mathrm{z} \in \mathrm{f}^{-1}\left(\mathrm{x}_{1}+\mathrm{y}_{1}\right)
$$$$
\geq \mathrm{T}\left\{\mathrm{A}\left(\mathrm{x}_{0}\right), \quad \mathrm{A}\left(\mathrm{y}_{\mathrm{o}} \geq \mathrm{T} \quad \text { Sup } \mathrm{A}(\mathrm{h})\right.\right.
$$

Sup A(h)

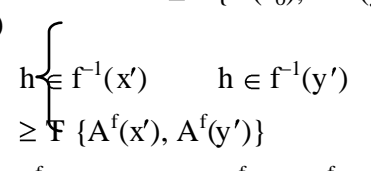

$A^{f}\left(x^{\prime}+y^{\prime}\right) \geq T\left\{A^{f}\left(x^{\prime}\right), A^{f}\left(y^{\prime}\right)\right\}$

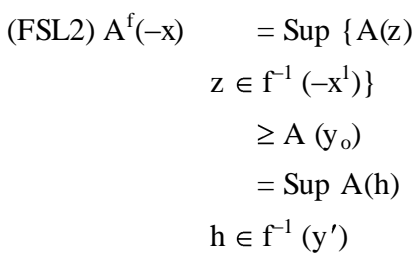
Definition (2.4)

$=\mathrm{A}^{\mathrm{f}}\left(\mathrm{y}^{\prime}\right) \cdot$ Similarly, we can prove $($ FSL3), (FSL4) of

Preposition 3.10 : Let $f: G \rightarrow G^{\prime}$ be a Lattice group homomorphism and A be a Fuzzy Soft Lattice of $\mathrm{G}^{\prime}$ then $\mathrm{f}^{-}$ ${ }^{1}(\mathrm{~A})$ is a Fuzzy Soft Lattice of G.

Proof : Let $x, y \in G$ and $A$ is a Fuzzy Soft Lattice of $G^{\prime}$.

$$
\begin{aligned}
& (\mathrm{FSL} 1) \mathrm{f}^{-1}(\mathrm{~A})(\mathrm{x}+\mathrm{y}) \quad=\operatorname{A~f}(\mathrm{x}+\mathrm{y}) \\
& =A(f(x)+f(y)) \\
& \geq \mathrm{T}\{\mathrm{A}(\mathrm{f}(\mathrm{x})+\mathrm{f}(\mathrm{y})\} \\
& \geq \mathrm{T}\left\{\mathrm{f}^{-1}(\mathrm{~A})(\mathrm{x}), \mathrm{f}^{-1}(\mathrm{~A})(\mathrm{y})\right\} \\
& \left(\text { FSL2) } f^{-1}(\text { A) }(-x)=\operatorname{A~} f(-x)\right. \\
& \text { (FSL3) } \mathrm{f}^{-1}(\mathrm{~A})(\mathrm{x} \mathrm{V} y) \\
& \geq \mathrm{A} \mathrm{f}(\mathrm{x}) \\
& \geq \mathrm{f}^{-1}(\mathrm{~A})(\mathrm{x}) \\
& =\mathrm{Af}(\mathrm{x} V \mathrm{y}) \\
& =A(f(x) V f(y)) \\
& \geq \mathrm{T}\{\mathrm{A} \mathrm{f}(\mathrm{x}), \mathrm{A} \mathrm{f}(\mathrm{y}))\} \\
& \geq \mathrm{T}\left\{\mathrm{f}^{-1}(\mathrm{~A})(\mathrm{x}), \mathrm{f}^{-1}(\mathrm{~A})(\mathrm{y})\right\} \\
& (\mathrm{FSL} 4) \mathrm{f}^{-1}(\mathrm{~A})(\mathrm{x} \Lambda \mathrm{y}) \quad=\mathrm{Af}(\mathrm{x} \Lambda \mathrm{y}) \\
& =A(f(x) \Lambda f(y)) \\
& \geq \mathrm{T}\{\text { A f }(\mathrm{x}), \operatorname{A~f}(\mathrm{y}))\} \\
& \geq \mathrm{T}\left\{\mathrm{f}^{-1}(\mathrm{~A})(\mathrm{x}), \mathrm{f}^{-1}(\mathrm{~A})(\mathrm{y})\right\}
\end{aligned}
$$

$\therefore \mathrm{f}^{-1}(\mathrm{~A})$ is a Fuzzy Soft Lattice of $\mathrm{G}$.

Proposition 3.11 : Let A be a fuzzy normal soft lattice of G.Then for all $x, y \in G . A([x, y])=A(0))$.

\section{Proof :}

Since A is fuzzy normal soft lattice of G. Then we have

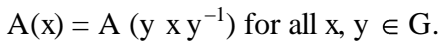

Replacing $\mathrm{x}$ by $\mathrm{x}^{-1}$ and $\mathrm{y}$ by $\mathrm{y}^{-1}$, it gives

$\mathrm{A}\left(\mathrm{y}^{-1}\right)=\mathrm{A}\left(\mathrm{x}^{-1} \mathrm{y}^{-1} \mathrm{xy}\right)$ or $\mathrm{A}\left(\mathrm{x}^{-1} \mathrm{y}^{-1} \mathrm{x} \mathrm{yy}^{-1}\right)=\mathrm{A}\left(\mathrm{y}^{-1}\right)$ or $\mathrm{A}\left([\mathrm{x}, \mathrm{y}] \mathrm{y}^{-1}\right)$

$$
=\mathrm{A}\left(\mathrm{y}^{-1}\right) \text { or } \mathrm{A}[\mathrm{x}, \mathrm{y}]=\mathrm{A}(0)
$$




\section{4 : Direct product of Fuzzy Soft Lattices}

Definition 4:1 : Let $A_{i}$ be a Fuzzy Soft Lattice of $G_{i}$, for $i$ $=1,2,3 \ldots \mathrm{n}$. Than the product of $\mathrm{A}_{\mathrm{i}}(\mathrm{i}=1,2,3, \ldots \mathrm{n})$ is the function $A_{1} \times A_{2} \times A_{3} \times \ldots \times A_{n}: G_{1} \times G_{2} \times \ldots \times G_{n} \rightarrow L$ defined by

$\left(A_{1} \times A_{2} \times A_{3} \times \ldots \times A_{n}\right)\left(x_{1}, x_{2}, \ldots x_{n}\right)=T\left\{A_{1}\left(x_{1}\right), A_{2}\left(x_{2}\right) \ldots\right.$ $\left.A_{n}\left(x_{n}\right)\right\}$

Proposition 4.2 : The Direct product of Fuzzy Soft Lattices is a Fuzzy Soft Lattices.

Proof :

Let $\mathrm{x}=\left(\mathrm{x}_{1}, \mathrm{x}_{2}, \ldots \mathrm{x}_{\mathrm{n}}\right), \mathrm{y}=\left(\mathrm{y}_{1}, \mathrm{y}_{2}, \ldots \mathrm{y}_{\mathrm{n}}\right)$

Let $\left(x_{1}, x_{2}, \ldots, x_{n}\right),\left(y_{1}, y_{2}, \ldots y_{n}\right) \in A_{1} x_{2} x_{2} A_{3} x \ldots A_{n}=$ $\mathrm{f}_{\mathrm{a}}$

$\left(\right.$ FSL1) $f_{a}(x+y)=f_{a}\left(\left(x_{1}, x_{2}, \ldots, x_{n}\right)+\left(y_{1}, y_{2}, \ldots y_{n}\right)\right)$

$=A_{1} \times A_{2} \times A_{3} \times \ldots \times A_{n}\left(\left(x_{1}+y_{1}\right),\left(x_{2}+y_{2}\right) \ldots\left(x_{n}+y_{n}\right)\right)$

$=\mathrm{f}_{\mathrm{a}}\left(\left(\mathrm{x}_{1}+\mathrm{y}_{1}\right),\left(\mathrm{x}_{2}+\mathrm{y}_{2}\right), \ldots,\left(\mathrm{x}_{\mathrm{n}}+\mathrm{y}_{\mathrm{n}}\right)\right)$

$\geq \mathrm{T}\left\{\mathrm{A}_{1}\left(\mathrm{x}_{1}+\mathrm{y}_{1}\right), \mathrm{A}_{2}\left(\mathrm{x}_{2}+\mathrm{y}_{2}\right), \mathrm{A}_{\mathrm{n}}\left(\mathrm{x}_{\mathrm{n}}+\mathrm{y}_{\mathrm{n}}\right)\right\}$

$\geq \mathrm{T}\left\{\mathrm{T}\left\{\mathrm{A}_{1}\left(\mathrm{x}_{1}\right), \mathrm{A}_{1}\left(\mathrm{y}_{1}\right)\right\}, \mathrm{T}\left\{\mathrm{A}_{2}\left(\mathrm{x}_{2}\right), \mathrm{A}_{2}\left(\mathrm{y}_{2}\right)\right\}, \ldots \mathrm{T}\left\{\mathrm{A}_{\mathrm{n}}\left(\mathrm{x}_{\mathrm{n}}\right), \mathrm{A}_{\mathrm{n}}\left(\mathrm{y}_{\mathrm{n}}\right)\right\}\right.$, \}

$\geq \mathrm{T}\left\{\mathrm{T}\left\{\mathrm{A}_{1}\left(\mathrm{x}_{1}\right), \mathrm{A}_{2}\left(\mathrm{x}_{2}\right), \ldots, \mathrm{A}_{\mathrm{n}}\left(\mathrm{x}_{\mathrm{n}}\right)\right\}, \mathrm{T}\left\{\mathrm{A}_{1}\left(\mathrm{y}_{1}\right), \mathrm{A}_{2}\left(\mathrm{y}_{2}\right) \ldots\right.\right.$, $\left.\mathrm{A}_{\mathrm{n}}\left(\mathrm{y}_{\mathrm{n}}\right)\right\}$

$\geq \mathrm{T}\left\{\left(\mathrm{A}_{1} \times \mathrm{A}_{2} \times \ldots \times \mathrm{A}_{\mathrm{n}}\right)\left(\mathrm{x}_{1}, \mathrm{x}_{2}, \ldots, \mathrm{x}_{\mathrm{n}}\right),\left(\mathrm{A}_{1} \times \mathrm{A}_{2} \times \ldots \times \mathrm{A}_{\mathrm{n}}\right)\right.$ $\left.\left(\mathrm{y}_{1}, \mathrm{y}_{2}, \ldots, \mathrm{y}_{\mathrm{n}}\right)\right\}$

$\geq \mathrm{T}\{\lambda(\mathrm{x}), \lambda(\mathrm{y})\}$

(FSL2) $\mathrm{f}_{\mathrm{a}}(-\mathrm{x}) \quad=\mathrm{f}_{\mathrm{a}}(-\mathrm{x})$

$=\left(A_{1} \times A_{2} \times \ldots \times A_{n}\right)\left(\left(-x_{1}\right),\left(-x_{2}\right), \ldots,\left(-x_{n}\right)\right)$

$=\mathrm{T}\left\{\mathrm{A}_{1}\left(-\mathrm{x}_{1}\right), \mathrm{A}_{2}\left(-\mathrm{x}_{2}\right), \ldots \mathrm{A}_{\mathrm{n}}\left(-\mathrm{x}_{\mathrm{n}}\right)\right\}$

$\geq \mathrm{T}\left\{\mathrm{A}_{1}\left(\mathrm{x}_{1}\right), \mathrm{A}_{2}\left(\mathrm{x}_{2}\right) \ldots \mathrm{A}_{\mathrm{n}}\left(\mathrm{x}_{\mathrm{n}}\right)\right\}$

$\geq\left(\mathrm{A}_{1} \times \mathrm{A}_{2} \mathrm{x} \ldots \mathrm{x} \mathrm{A}_{\mathrm{n}}\right)\left(\mathrm{x}_{1}, \mathrm{x}_{2}, \ldots, \mathrm{x}_{\mathrm{n}}\right)$

$\geq \mathrm{f}_{\mathrm{a}}(\mathrm{x})$

$\left(\right.$ FSL3) $f_{a}(x V y)=f_{a}\left(\left(x_{1}, x_{2}, \ldots x_{n}\right) V\left(y_{1}, y_{2}, \ldots y_{n}\right)\right)$

$=\mathrm{f}_{\mathrm{a}}\left(\mathrm{x}_{1} V \mathrm{y}_{1}, \mathrm{x}_{2} V \mathrm{y}_{2}, \ldots \mathrm{x}_{\mathrm{n}} \mathrm{Vy_{ \textrm {n } }}\right)$

$=\left(A_{1} \times A_{2} \times \ldots x A_{n}\right)\left(x_{1} V y_{1}, x_{2} V y_{2}, \ldots x_{n} V y_{n}\right)$

$=\mathrm{T}\left\{\mathrm{A}_{1}\left(\mathrm{x}_{1} \mathrm{Vy_{1 }}\right), \mathrm{A}_{2}\left(\mathrm{x}_{\mathrm{x}} \mathrm{V} \mathrm{y}_{2}\right), \ldots, \mathrm{A}_{\mathrm{n}}\left(\mathrm{x}_{\mathrm{n}} \mathrm{V} \mathrm{y}_{\mathrm{n}}\right)\right\}$

$\geq \mathrm{T}\left\{\mathrm{T}\left\{\mathrm{A}_{1}\left(\mathrm{x}_{1}\right), \mathrm{A}_{1}\left(\mathrm{y}_{1}\right)\right\}, \mathrm{T}\left\{\mathrm{A}_{2}\left(\mathrm{x}_{2}\right), \mathrm{A}_{2}\left(\mathrm{y}_{2}\right)\right\}, \quad \ldots, \mathrm{T}\left\{\mathrm{A}_{\mathrm{n}}\left(\mathrm{x}_{\mathrm{n}}\right)\right.\right.$, $\left.\left.\mathrm{A}_{\mathrm{n}}\left(\mathrm{y}_{\mathrm{n}}\right)\right\}\right\}$

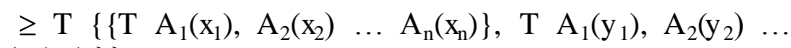
$\left.\left.\mathrm{A}_{\mathrm{n}}\left(\mathrm{y}_{\mathrm{n}}\right)\right\}\right\}$

$\geq T\left\{\left(A_{1} \times A_{2} \times \ldots \times A_{n}\right)\left(x_{1}, x_{2}, \ldots, x_{n}\right),\left(A_{1} \times A_{2} \times \ldots \times A_{n}\right)\right.$ $\left.\left(\mathrm{y}_{1}, \mathrm{y}_{2}, \ldots, \mathrm{y}_{\mathrm{n}}\right)\right\}$

$\geq \mathrm{T}\left\{\mathrm{f}_{\mathrm{a}}(\mathrm{x}), \mathrm{f}_{\mathrm{a}}(\mathrm{y})\right\}$.Similarly, we can show (FSL4) of definition (2.4)

Proposition 4.3 :The intersection of two Fuzzy Soft Lattices is a Fuzzy Soft Lattice.

Proof : Since A and B are Fuzzy Soft Lattices in G.

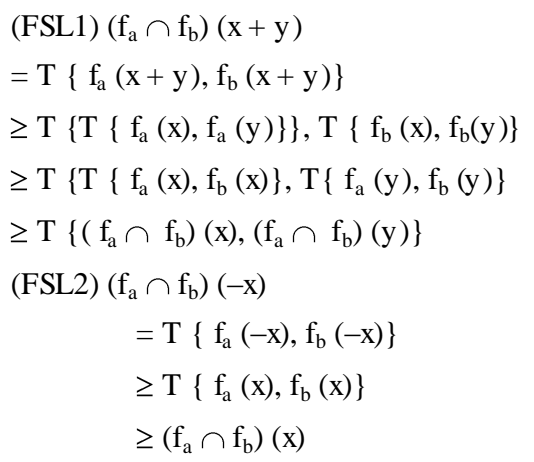

(FSL3) $\left(f_{a} \cap f_{b}\right)(x \vee y)$

$=\mathrm{T}\left\{\mathrm{f}_{\mathrm{a}}(\mathrm{x} V \mathrm{y}), \mathrm{f}_{\mathrm{b}}(\mathrm{x} V \mathrm{y})\right\}$

$\geq \mathrm{T}\left\{\mathrm{T}\left\{\mathrm{f}_{\mathrm{a}}(\mathrm{x}), \mathrm{f}_{\mathrm{b}}(\mathrm{y})\right\}, \mathrm{T}\left\{\mathrm{f}_{\mathrm{b}}(\mathrm{x}), \mathrm{f}_{\mathrm{b}}(\mathrm{y})\right\}\right\}$

$\geq \mathrm{T}\left\{\mathrm{T}\left\{\mathrm{f}_{\mathrm{a}}(\mathrm{x}), \mathrm{f}_{\mathrm{b}}(\mathrm{x})\right\}, \mathrm{T}\left\{\mathrm{f}_{\mathrm{a}}(\mathrm{y}), \mathrm{f}_{\mathrm{b}}(\mathrm{y})\right\}\right\}$

$\geq \mathrm{T}\left\{\left(\mathrm{f}_{\mathrm{a}} \cap \mathrm{f}_{\mathrm{b}}\right)(\mathrm{x}),\left(\mathrm{f}_{\mathrm{a}} \cap \mathrm{f}_{\mathrm{b}}\right)(\mathrm{y})\right\}$

Similarly, we can show (FSL4 of Definition (2.4)

Proposition 4.4: If $f_{a}$ is a Fuzzy Soft Lattice then $f_{a}^{c}$ is also a Fuzzy Soft Lattice.

Proof : For any $x, y \in G$.

(FSL1) $\mathrm{f}_{\mathrm{a}}^{\mathrm{c}}(\mathrm{x}+\mathrm{y})=1-\mathrm{f}_{\mathrm{a}}(\mathrm{x}+\mathrm{y})$

$$
\begin{aligned}
& \leq \mathrm{S}\left\{1-\mathrm{f}_{\mathrm{a}}(\mathrm{x}), 1-\mathrm{f}_{\mathrm{a}}(\mathrm{y})\right\} \\
& \leq \mathrm{S}\left\{\mathrm{f}_{\mathrm{a}}^{\mathrm{c}}(\mathrm{x}), \mathrm{f}_{\mathrm{a}}^{\mathrm{c}}(\mathrm{y})\right\}
\end{aligned}
$$

(FSL2) $\mathrm{f}_{\mathrm{a}}^{\mathrm{c}}(-\mathrm{x})=1-\mathrm{f}_{\mathrm{a}}(-\mathrm{x})$

$$
\begin{aligned}
& \leq 1-f_{a}(x) \\
& \leq f_{a}^{c}(x)
\end{aligned}
$$

(FSL3) $f_{a}^{c}(x \vee y)=1-f_{a}(x V y)$

$$
\begin{aligned}
& \leq \mathrm{S}\left\{1-\mathrm{f}_{\mathrm{a}}(\mathrm{x}), 1-\mathrm{f}_{\mathrm{a}}(\mathrm{y})\right\} \\
& \leq \mathrm{S}\left\{\mathrm{f}_{\mathrm{a}}^{\mathrm{c}}(\mathrm{x}), \mathrm{f}_{\mathrm{a}}^{\mathrm{c}}(\mathrm{y})\right\}
\end{aligned}
$$

(FSL4) $\mathrm{f}_{\mathrm{a}}^{\mathrm{c}}(\mathrm{x} \Lambda \mathrm{y})=1-\mathrm{f}_{\mathrm{a}}(\mathrm{x} \Lambda \mathrm{y})$

$$
\begin{aligned}
& \leq \mathrm{S}\left\{1-\mathrm{f}_{\mathrm{a}}(\mathrm{x}), 1-\mathrm{f}_{\mathrm{a}}(\mathrm{y})\right\} \\
& \leq \mathrm{S}\left\{\mathrm{f}_{\mathrm{a}}^{\mathrm{c}}(\mathrm{x}), \mathrm{f}_{\mathrm{a}}^{\mathrm{c}}(\mathrm{y})\right\} . \text { Hence } \mathrm{f}_{\mathrm{a}}^{\mathrm{c}} \text { is fuzzy }
\end{aligned}
$$

soft lattice.

\section{CONCLUSION}

FULI [3] introduced the concept of soft lattices and P.K.. Maji et. al [6] investigated the concept of fuzzy soft sets (2001). In this paper we introduce the notion of fuzzy soft lattices with respect to soft lattice $\mathrm{L}$ under the triangular norms. To extend this work on could study the properties of fuzzy soft sets, in other algebraic structures such as soft G-modules and fields.

\section{APPLICATIONS}

A Lattice structure has been found to be extremely important in the areas of Medical sciences, social sciences, environmental sciences, economics, and decision making problems. Also, it will be very useful to make all types of mathematical modeling. 


\section{ACKNOWLEDGEMENTS}

The authors are highly grateful to the referees for their valuable comments and suggestions for improving the paper.

\section{REFERENCES}

[1] H. Aktas, N. Cagman, Soft Sets and Soft groups, Information Sciences, 177 (2007) 2726-2735.

[2] A. Aygunoglu, H. Aygun, Introduction to fuzzy soft groups, Computer Math. Appl. 58 (2009) 1279-1286.

[3] FULI, Soft Lattices, Global Journal of Science frontier research, Vol. 10, issue 4 (2010), pp. 56-58.

[4] F. Feng, Y.B. Jun and X. Zhao, Soft Semirings, Computers and Mathematics with Applications 56 (2008) 2621-2628.
[5] J.L. Liu and R.X. Yan, Fuzzy Soft Sets and Fuzzy Soft Groups, Chinese Control and decision Conference, Guilin, China (2008)

[6] P.K. Maji, R. Biswas and A.R. Roy, Fuzzy Soft Sets, Journal of Fuzzy Math. 9(3) (2001) 589-602.

[7] P.K. Maji, R. Biswas and A.R. Roy, Soft set theory, Computers Math. Applic. 45 (2003) 555-562

[8] M. Marudai, V. Rajendran, Characterization of fuzzy lattices on a group with respect to T-norms, International Journal of Computer Applications (0975-8887). Volume 8 - No.8 (2010) pp 8-14.

[9] D. Molodtsov, Soft set theory first results, Computers Math. Appl. 37 (4/5) (1999) pp. 19-31.

[10] A. Rosenfeld, Fuzzy groups, J. Math, Anal. Appl.35 (1971) $512-517$

[11] L.A. Zadeh, Fuzzy sets, Information and Control. 8, (1965) $338-353$. 